\title{
A influência do board interlocks na prática da divulgação voluntária em empresas brasileiras
}

\author{
The influence of board interlocks on the practice of voluntary disclosure in Brazilian \\ companies
}

\section{La influencia del board interlocks en la práctica de la divulgación voluntaria en empresas brasileñas}

\section{Flávio Ribeiro}

Mestre em Contabilidade pela Universidade Federal do Paraná (UFPR)

Professor do Departamento de Ciências Contábeis da Universidade Estadual do Centro Oeste (Unicentro)

Endereço: Av. Prefeito Lothário Meissner, 632 - Campus III - Jardim Botânico

CEP: 80210-070 - Curitiba/PR - Brasil

E-mail: flavioribeiro@unicentro.br

Telefone: (41) 3360-4193

\section{Silvia Consoni}

Mestre em Contabilidade pela Universidade Federal do Paraná (UFPR)

Professora do Departamento de Ciências Contábeis da Universidade Federal do Paraná (UFPR)

Endereço: Av. Prefeito Lothário Meissner, 632 - Campus III - Jardim Botânico

CEP: 80210-070 - Curitiba/PR - Brasil

E-mail: silviaconsoni@yahoo.com.br

Telefone: (41) 3360-4193

\section{Romualdo Douglas Colauto}

Pós-Doutor em Contabilidade pela Universidade de São Paulo (USP)

Doutor em Engenharia de Produção pela Universidade Federal de Santa Catarina (UFSC)

Professor do Programa de Pós-Graduação em Ciências Contábeis da Universidade Federal do Paraná (UFPR)

Endereço: Av. Prefeito Lothário Meissner, 632 - Campus III - Jardim Botânico

CEP: 80210-070 - Curitiba/PR - Brasil

E-mail: rdcolauto.ufpr@gmail.com

Telefone: (41) 3360-4193

Artigo recebido em 05/01/2018. Revisado por pares em 26/03/2018. Reformulado em 20/03/2019. Recomendado para publicação em 20/03/2019 por Carlos Eduardo Facin Lavarda Editor-Chefe). Publicado em 10/04/2019. 


\section{Resumo}

O estudo investiga a influência da estrutura de formação do board interlocks na divulgação voluntária de empresas de capital aberto no Brasil. Utiliza-se a abordagem de análise de redes sociais para captar e avaliar o nível de relacionamento existente entre empresas em função dos laços formados por membros do conselho de administração. Duas hipóteses exploram as medidas de (i) centralidade de grau (degree centrality) e de (ii) centralidade de intermediação (betweenness centrality), as quais têm potencial de descrever o posicionamento dos atores em uma rede. A terceira hipótese endereça a questão da qualidade dos laços (holes). Os resultados sugerem que a estrutura de formação do board interlocks aumenta a propensão de as empresas reportarem informações de natureza voluntária em seus relatórios anuais. Interpreta-se, por isso, que o board interlocks atua como um fator que favorece a disseminação de práticas de divulgação voluntária.

Palavras-chave: Board Interlocks; Divulgação voluntária; Análise de redes sociais

\section{Abstract}

The study investigates the influence of the board interlocks structure in the voluntary disclosure of publicly traded companies in Brazil. The social network analysis approach is used to capture and evaluate the level of relationship existing between companies due to the ties formed by members of the board of directors. Two hypotheses explore the measures of (i) degree centrality and (ii) betweenness centrality, which have the potential to describe the positioning of the actors in a network. The third hypothesis addresses the issue of bond quality (holes). The results suggest that the board structure of board interlocks increases the propensity of companies to report information of a voluntary nature in their annual reports. It is interpreted, therefore, that the board interlocks acts as a factor that favors the dissemination of practices of voluntary disclosure.

Keywords: Board Interlocks; Voluntary disclosure; Analysis of social networks

\section{Resumen}

El estudio investiga la influencia de la estructura de formación del board interlocks en la divulgación voluntaria de empresas de capital abierto en Brasil. Se utiliza el enfoque de análisis de redes sociales para captar y evaluar el nivel de relación existente entre empresas en función de los vínculos formados por miembros del consejo de administración. Dos hipótesis explotan las medidas de (i) centralidad de grado (degree centralidad) y de (ii) centralidad de intermediación (betweenness centrality), las cuales tienen potencial de describir el posicionamiento de los actores en una red. La tercera hipótesis aborda la cuestión de la calidad de los lazos (holes). Los resultados sugieren que la estructura de formación del board interlocks aumenta la propensión de que las empresas reporten información de carácter voluntario en sus informes anuales. Se interpreta, por eso, que el board interlocks actúa como un factor que favorece la diseminación de prácticas de divulgación voluntaria.

Palabras clave: Board Interlocks; Divulgación voluntaria; Análisis de redes sociales

\section{Introdução}

As empresas têm compartilhado membros do conselho de administração, fenômeno denominado board interlocks (MIZRUCHI, 1996), para estabelecerem conexões que são comumente caracterizadas como mecanismos para a disseminação de estratégias e comportamentos semelhantes (HAUNSCHILD; BECKMAN, 1998; CARPENTER; WESTPHAL, 2001; HILLMAN; CANNELLA; PAETZOLD, 2000; WESTPHAL; SEIDEL; 
STEWART, 2001), embora nem sempre destinados à manutenção da adequada governança corporativa (FICH; WHITE, 2005; FICH; SHIVDASANI, 2006).

Existem diferentes orientações teóricas para a análise da natureza e funcionamento dos conselhos de administração (HUNG, 1998). Hillman e Dalziel (2003) propõem que as funções de monitoramento e de fornecimento de recursos podem ser analisadas de forma integrada. $\mathrm{O}$ fundamento teórico da função de monitoramento e supervisão do conselho de administração deriva da Teoria da Agência em que a eficácia do acompanhamento realizado por esse conselho seria determinada, entre outros aspectos, pela sua composição no que se refere à independêcia como um fator positivo e a dualidade como um fator negativo (FICH; WHITE, 2005; ONG; WAN, 2001). Na perspectiva da Teoria da Dependência de Recursos (PFEFFER; SALANCIK, 1978), o papel do conselho de administração é o de fornecer acesso a recursos críticos (materiais e simbólicos) e oportunizar conexões entre empresas para construir redes de cooperação que facilitariam a racionalização sobre incertezas (DALZIEL; GENTRY; BOWERMAN, 2011; HAYNES; HILLMAN, 2010).

Gibbins, Richardson e Waterhouse (1990) explicitam que a divulgação voluntária é considerada elemento de estratégia de comunicação das empresas e, assim, as informações corporativas são reconhecidas como recursos de consequências econômicas incertas. Por exemplo, um dos incentivos para a divulgação voluntária em empresas que dependem fortemente de fontes de financiamento externo, dentre outros, é a redução do custo de capital (BEYER et al., 2010), porém, determinados conteúdos de divulgação voluntária têm potencial de comprometer a vantagem competitiva das empresas (WAGENHOFER, 1990).

Membros do conselho que atuam em mais uma empresa podem analisar o ambiente de negócios e usar sua experiência em muitos conselhos corporativos para lidar com a incerteza associada a decisões estratégicas (PFEFFER; SALANCIK, 1978; AU; PENG; WANG, 2000). Nesse sentido, o conselho de administração pode interferir na determinação do mix específico de divulgação corporativa (CORE, 2001; CHENG; COURTENAY, 2006; GARCÍA-MECA; SÁNCHEZ-BALLESTA, 2010) e, práticas de divulgação voluntária são compartilhadas e, até mesmo, moldadas pelo board interlocks (BRAAM; BORGHANS, 2014, CAI et al., 2014; CHAN et al., 2017). Ainda, a Comissão de Valores Mobiliários (CVM, 2002) e o Instituto Brasileiro de Governança Corporativa (IBGC, 2015) expressam que, dentre as responsabilidade dos membros do conselho de administração, inclui-se a supervisão do processo de geração e de divulgação de informações contábeis ao mercado de capitais; o que não exclui a definição de uma política de divulgação voluntária nas empresas.

O board interlocks tende a formar uma rede de conexões e, como descrito por Connelly e Slyke (2012), os atores (indivíduos, empresas) de uma rede não estão exatamente na mesma posição. Isto é explicitado, também, por Hauschild e Beckman (1998), ao provocarem a discussão sobre a ideia de que os interlocks não desempenham influência uniforme. Nesse sentido, diferentes tipos de laços têm diferentes efeitos, ou seja, a configuração estrutural das redes pode afetar o relacionamento intercorporativo (MIZRUCHI, 2006; GRANOVETTER, 1973), o que traz à discussão a questão de os atributos de redes exercerem influência no fluxo de recursos informacionais. Por conseguinte, o objetivo delineado é o de investigar a influência da estrutura de formação do board interlocks na divulgação voluntária de empresas de capital aberto no Brasil no período de 2010 a 2015.

Embora existam evidências de que o provimento de informações com base em medidas financeiras e não financeiras (BRAAM; BORGHANS, 2014), a realização de teleconferências (CHAN et al., 2017) e a interrupção do guindance trimestral (CAI et al., 2014), sejam afetadas pelo board interlocks, em tais estudos, o board interlocks não é caracterizado pelas estruturas de redes formadas, mas apenas pela presença desse tipo de conexão. No entanto, diferentemente dessas pesquisas, utiliza-se a abordagem de análise de redes sociais (WASSERMAN; FAUST, 1994) para, assim, captar e avaliar o nível de relacionamento existente entre empresas em 
função dos laços formados por membros dos conselhos de administração. Duas hipóteses exploram as medidas de (i) centralidade de grau (degree centrality) e de (ii) centralidade de intermediação (betweenness centrality), as quais têm potencial de descrever o posicionamento dos atores em uma rede (WASSERMAN; FAUST, 1994). A terceira hipótese endereça a questão da qualidade dos laços (structural holes), ou seja, ligações não redundantes, as quais proporcionariam acesso às fontes diversificadas de informação (BURT, 2004).

As empresas tendem a considerar, quando do estabelecimento de estratégias corporativas, o ambiente institucional no qual operam (MIZRUCHI; STEARNS, 1988; LAZZARINI, 2007; SUBRAHMANYAN, 2008; ROSSONI; MACHADO-DA-SILVA, 2013). Por exemplo, empresas que operam em mercados de capitais menos desenvolvidos têm poucos incentivos para o provimento de informações (LA PORTA; LOPEZ-DE-SILANES; VISHNY, 1998; BALL; ROBIN; WU, 2003; LEUZ; NANDA; WYSOCHI, 2003), e isto é o que se presume acontecer no mercado de capitais brasileiro (LEAL, OLIVEIRA, 2002; SILVEIRA et al., 2009;). Além disso, o modelo de governança corporativa adotado pelas empresas não é apenas uma resposta de equilíbrio ao ambiente legal em que as empresas atuam. Klapper e Love (2004) e Silveira et al. (2009) observaram uma grande variação na qualidade das práticas de governança corporativa das empresas submetidas ao mesmo ambiente contractual.

No mercado de capitais brasileiro a presença de grupos econômicos, de fundos de pensão, de controle governamental, além de profissionais com reconhecida experiência, são fatores que contribuem para a formação do board interlocks (RIBEIRO; COLAUTO, CLEMENTE, 2016), enquanto a concentração acionária em empresas familiares parece inibílo (SANTOS; SILVEIRA, 2007). Além disso, nesse mesmo ambiente institucional, nos anos de 2005 a 2012, as empresas aumentaram a presença de informações de natureza voluntária no Relatório da Administração e Formulário de Referência, embora de forma bastante heterogênea entre empresas e com fraca tendência de alteração do enfoque informacional (CONSONI; COLAUTO, 2016).

Em consonância com as expectativas gerais, os resultados obtidos indicaram que as empresas interligadas por meio dos membros do conselho de administração têm maior propensão de reportar informações econômico-financeiras de natureza voluntária no Relatório da Administração e Formulário de Referência. Interpreta-se, por isso, que a estrutura do board interlocks pode favorecer a disseminação da prática da divulgação voluntária, embora não se esteja especificamente considerando que o conteúdo informacional seja afetado de maneira uniforme pela posição que ocupam cada um dos conselheiros interligados. Portanto, esta é apenas uma evidência sobre um fator, de uma série de fatores potenciais que induzem as práticas de divulgação voluntária no mercado brasileiro, no qual os incentivos para a divulgação voluntária parecem ser relativamente fracos.

\section{Revisão da literatura e desenvolvimento de hipóteses}

A divulgação de informações corporativas tem potencial de mitigar a assimetria informacional entre provedores de recursos e seus tomadores (HEALY; PALEPU, 2001). As informações são propagadas por meio de dois canais de comunicação distintos, isto é, um compulsório e outro voluntário, cada qual com uma dinâmica bastante particular. A divulgação compulsória restringe-se ao conteúdo mínimo que é requerido em leis e regulamentos em geral, enquanto a divulgação voluntária refere-se à informação adicional que é liberada por livre escolha (DEPOERS, 2000) e, por isso, considerada elemento da estratégia de comunicação das empresas (GIBBINS; RICHARDSON; WATERHOUSE, 1990).

A forma como uma empresa estrutura sua política de comunicação voluntária depende dos custos e benefícios percebidos sendo, assim, deixada à discricionariedade da administração (DYE, 2001). Por isso, existem diferentes incentivos que levam as empresas à prática da 
divulgação voluntária como, por exemplo, para distinguir a sua performance em relação às demais empresas, reduzir o custo de captação de recursos financeiros e, também, permitir que os gestores demonstrem o uso dos recursos no melhor interesse dos acionistas (BERTOMEU; BEYER; DYE, 2011; BEYER et al., 2010; CAMPBELL; SHRIVES; BOHMBACHSAAGAR, 2001; CORE, 2001; HEALY; PALEPU, 2001). É concebível, por isso, que os incentivos para o provimento voluntário de informações dependa das características das empresas (MURCIA; SANTOS, 2012; BRANDÃO et al., 2013), dos custos (proprietary costs) de revelar informações à concorrência (DYE, 1986; WAGENHOFER, 1990) e de outras pressões (AMBROZINI, 2017) que provocam, assim, variação na divulgação voluntária entre empresas, como ocorre no mercado brasileiro (CONSONI; COLAUTO, 2016), mas, também, entre países em resposta às pressões do ambiente institucional em que as empresas operam (BALL; ROBIN; WU, 2003).

Para responder adequadamente às expectativas dos provedores de capital e de outras potenciais fontes de pressão externa, cabe ao conselho de administração, entre outras funções, monitorar as ações dos gestores, fixar diretrizes da estratégia empresarial, ratificar decisões relevantes e assegurar a confiabilidade das informações divulgadas (ADAMS; HERMALIN; WEISBACH, 2010; ANDERSON; MANSI; REEB, 2004; MARTINS; RODRIGUES, 2005). A função de monitoramento e supervisão desempenhada pelo conselho de administração está, segundo Hillman e Dalziel (2003), integrada à função de fornecimento de recursos, a qual é descrita pela Teoria da Dependência de Recursos.

A Teoria da Dependência de Recursos (PFEFFER; SALANCIK, 1978) pressupõe que as empresas individualmente não são capazes de controlar inteiramente todas as condições necessárias para atingir êxito nas ações que empreendem e, segundo Hung (1998), Hillman e Dalziel (2003), Hillman, Withers e Collins (2009), essa teoria, ao se concentrar nas ações para a redução de dependências externas, elucida que o board interlocks é o resultado da aplicação da estratégia de cooptação ou de persuasão de membros do conselho e funciona como um fator crítico para a aquisição e manutenção de recursos adequados, incluindo recursos financeiros, físicos e de informação. Como as empresas estão sujeitas a restrições de recursos do ambiente externo enfatiza-se que relações de trocas são estabelecidas com outras empresas para formar coalisões mutuamente benéficas. Tais coalisões garantiriam a adaptação ao ambiente no qual operam, o gerenciamento e o controle do fluxo de recursos materiais e simbólicos, ou seja, membros do conselho de administração quando interligados são potencialmente capazes de fornecer à empresa acesso a recursos externos, os quais, de outra forma, não estariam disponíveis.

As conexões formadas entre conselhos de administração, segundo Mizruchi e Stearns (1988), são a expressão da necessidade de as empresas recrutarem profissionais de boa reputação e prestígio no ofício de conselheiro e, assim, promover a propagação de informações e práticas de interesse corporativo. Profissionais de um conselho de administração atuando em conselhos de administração de outras empresas oportunizam a identificação e, consequentemente, o compartilhamento de diversas práticas corporativas (HAUSCHILD, 1993; HAUSCHILD; BECKMAN, 1998; BECKMAN, HAUSNCHILD, PHILIPS, 2004; STUART, YIM, 2010; ROSSONI; MACHADO-DA-SILVA, 2012; BRAAM; BORGHANS, 2014, CAI et al., 2014; RIBEIRO; COLAUTO, 2016; CHAN et al., 2017). Para Hillman, Cannella e Paetzold (2000) conselheiros interligados não só observam diversas práticas corporativas como, também, testemunham de forma direta seus desdobramentos e, por isso, possuem poder de persuasão sobre os demais membros do conselho exercendo influência na disseminação de práticas potencialmente efetivas.

As conclusões de Cai et al. (2014) são consistentes com a noção de que os diretores interligados servem como canais de compartilhamento de informações, o que pode resultar na mudança de políticas de divulgação corporativa devido a baixa ambiguidade nas ações e 
incertezas quanto aos resultados ou consequências dessas mesmas ações. Isto porque, os autores identificaram que diretores interligados são mais propensos a cessação da divulgação do guindance trimestral, em especial, se a experiência anterior de cessação provou ser bem sucedida em alguma das empresas conectadas.

Nessa mesma linha, Braam e Borghans (2014) encontraram evidências de que a presença de laços de interligação entre membros do conselho de administração e auditores externos favoreceu a divulgação voluntária de medidas financeiras e não financeiras de performance em uma amostra de 149 empresas holandesas com títulos listados no ano de 2004 na New York Stock Exchange (NYSE). O estudo de Chan et al. (2017) revelou que o board interlocks aumenta a probabilidade de realização e frequência de teleconferências, proxy de divulgação voluntária, sendo esta evidência mais pronunciada se as conexões forem mantidas por meio de diretores independentes e entre empresas com maior assimetria de informações.

Na identificação do board interlocks Cai et al. (2014), Braam e Borghans (2014) e Chan et al. (2017) não caracterizaram as estruturas formadas, mas somente a presença de conexões. Os laços existentes entre conselheiros e, por conseguinte, entre empresas, tende a formar uma rede (conjunto de conexões entre elementos). As redes sociais são dinâmicas, o que significa dizer que laços entre elementos podem ser adicionados ou removidos ao longo do tempo (LAZZARINI, 2007). Segundo Connelly e Slyke (2012), a quantidade de laços e a posição que assumem os atores (indivíduos, empresas) determinam a estrutura de composição de uma rede, o que acaba moldando a maneira como as empresas funcionam.

Existem diferentes medidas para a análise da estrutura de composição das redes sociais, como medidas de centralidade e de configuração. Medidas de centralidade descrevem as localizações dos atores em termos de quão perto estão do "centro" da ação em uma rede e, podem ser definidas pelo número de ligações incidentes de um vértice (WASSERMAN; FAUST, 1994). Para abordar a maneira pela qual a estrutura de composição do board interlocks pode influenciar no fluxo de informação e, consequentemente, na prática da divulgação voluntária três hipóteses são estabelecidas. Duas hipóteses, nesse estudo, são elaboradas considerando a abordagem de centralidade das posições por meio da medida de centralidade de grau (degree centrality) e de centralidade de intermediação (betweenness centrality). A terceira hipótese endereça a questão da configuração da rede (BURT, 2004), utilizando-se a medida de structural holes para se referir à qualidade dos laços estabelecidos. O conceito de structural holes (BURT, 1980, 2004; GOYAL; VEGA-REDONDO, 2007) introduz a ideia de que os laços são estabelecidos para conectar diferentes clusters e, quando isso ocorre, maior será a probabilidade de as informações compartilhadas por esse canal serem originais e valiosas.

A centralidade de grau possibilita a avaliação dos laços diretos estabelecidos entre conselheiros e, pode ser interpretado como a probabilidade que o vértice tem de receber alguma informação da rede (MENDES-DA-SILVA et al., 2008). De acordo com Mizruchi (1996) os laços diretos (proximidade e quantidade de laços) tendem a minimizar as incertezas aliadas às escolhas estratégicas por proporcionar identificar práticas emergentes entre aqueles que os estabelecem. Entende-se, portanto, que sob tal condição, a posição das empresas na rede é mais favorável ou privilegiada quanto ao acesso a recursos distintos (BORGATTI; CROSS, 2003), pois facilitam a confiança e o intercâmbio cooperativo (COLEMAN, 1988) e, consequentemente, abre-se a oportunidade para a adoção de práticas similares de divulgação voluntária, conforme enunciado na seguinte hipótese:

$\mathrm{H}_{1}$ : A associação positiva entre o board interlocks e o índice de divulgação voluntária das empresas brasileiras de capital aberto depende da centralidade do grau (degree centrality).

A centralidade de intermediação quantifica o número de vezes que um vértice age como ponte ao longo do caminho mais curto entre dois outros vértices, ou seja, capta a presença de 
laços indiretos (WASSERMAN; FAUST, 1994; MENDES-DA-SILVA et al., 2008; ROSSONI; MACHADO-DA-SILVA, 2012). Essa medida permite avaliar a capacidade de a empresa negociar contatos, isto porque, endereça a suposição que, se determinada empresa deseja influenciar outra empresa utilizaria, para tanto, de um intermediário (BECKMAN; HAUSNCHILD; PHILIPS, 2004). Dessa forma, presume-se que a interação entre empresas defina uma escolha estratégica, a qual acaba por incentivar a adoção de práticas similares de divulgação voluntária, conforme enunciado na seguinte hipótese:

$\mathrm{H}_{2}$ : A associação positiva entre o board interlocks e o índice de divulgação voluntária das empresas brasileiras de capital aberto depende da centralidade de intermediação (betweenness centrality).

O compartilhamento de práticas corporativas entre empresas pode, também, ser influenciada pela qualidade dos laços formados (structural holes). A qualidade dos laços é delineada pelo estabelecimento de laços não redundantes, isto é, quando estes são estabelecidos entre diferentes clusters de forma a eliminar um "buraco" estrutural (CONNELY; SLYKE, 2012). O conceito de structural holes desenvolve a ideia delineada por Granoveter (1973) sobre laços fortes e laços fracos sob o fundamento de que há maior homogeneidade de informações e de comportamento dentro de qualquer grupo do que entre grupos. Assim, conselheiros que conectam empresas que já se apresentam conectadas estabelecem uma conexão redundante, a qual pode oferecer uma visão isolada das oportunidades e riscos associados às estratégias e aos usos de recursos potenciais.

O "buraco" em uma rede é, portanto, entendido como uma lacuna entre dois atores (indivíduos, empresas) que possuem fontes complementares de informação (BURT, 2004). Aquele que fecha um "buraco estrutural" (structural holes) torna-se essencial para conectar os demais (GOYAL; VEGA-REDONDO, 2007), e pode proporcionar acesso ou exposição a fontes diversificadas e oportunas de recursos informacionais e de conhecimento (CONNELY; SLYKE, 2012; WESTPHAL; SEIDEL; STEWART, 2001). Portanto, formula-se a seguinte hipótese de pesquisa:

$\mathrm{H}_{3}$ : A associação positiva entre o board interlocks e o índice de divulgação voluntária das empresas brasileiras de capital aberto depende da qualidade dos laços (structural holes).

\section{Procedimentos Metodológicos}

As variáveis do modelo estão representadas na equação (1), a qual explicita o entendimento de que o board interlocks, especificamente, pela estrutura das redes formadas, exerce influência nas práticas de divulgação voluntária das empresas.

$$
I D V_{\mathrm{i}, \mathrm{t}}=\beta_{1}+\text { Degree }_{\mathrm{i}, \mathrm{t}} \beta_{2}+\text { Betweeness }_{\mathrm{i}, \mathrm{t}} \beta_{3}+\text { Holes }_{\mathrm{i}, \mathrm{t}} \beta_{4}+\mathrm{ROA}_{\mathrm{i}, \mathrm{t}} \beta_{5}+\mathrm{ALAV}_{\mathrm{i}, \mathrm{t}} \beta_{6}+\varepsilon \mathrm{it}
$$

Em que,

IDV = índice de divulgação voluntária da empresa $i$ no ano $t$;

Degree $=$ centralidade de grau da empresa $i$ no ano $t$;

Betweeness $=$ centralidade de intermediação da empresa $i$ no ano $t$;

Holes $=$ qualidade dos laços da empresa $i$ no ano $t$;

$\mathrm{ROA}=$ razão entre o lucro líquido e o ativo total da empresa $i$ no ano $t$

ALAV $=$ razão entre as obrigações de curto e longo prazo e o ativo total da empresa $i$ no ano $t$; e $\varepsilon=$ termo de erro da empresa $i$ no ano $t$.

A variável dependente (IDV) é uma medida de incidência da divulgação voluntária nos relatórios anuais das empresas. Devido à ausência de uma medida de divulgação voluntária 
aceita unanimamente (BEYER et al., 2010), emprega-se o índice proposto por Consoni e Colauto (2016), o qual foi obtido pela verificação de 27 itens de divulgação que, em conjunto, referem-se às informações sobre mercado de atuação, estratégia corporativa, desempenho econômico-financeiro e aspectos operacionais. Para a codificação e a mensuração do IDV utilizou-se a metodologia de Consoni e Colauto (2016) atribuindo-se (i) zero ponto quando nenhuma informação é apresentada sobre o item em análise (informação ausente); (ii) um ponto quando a informação é apresentada em termos puramente descritivos (informação declarativa) e; (iii) dois pontos quando a informação é apresentada de forma declarativa e, também, expressa por números de natureza monetária e/ou não monetrária (informação declarativa e quantitativa). Dessa forma, o IDV de cada empresa foi calculado pela razão entre a pontuação individual obtida e a pontuação máxima ( 27 itens x 2 pontos) obtendo-se escores que variaram entre $0 \mathrm{e}$ 1 .

As variáveis independentes foram obtidas utilizando-se a técnica de análise de redes sociais (WASSERMAN; FAUST 1994), especificamente, duas medidas de centralidade de rede (degree centrality e betweeness centrality) e uma medida de configuração de rede (structural holes), o que permite explorar o fenômeno board interlocks na configuração do modelo em análise.

A variável de centralidade de grau (Degree) capta a centralidade local dos conselheiros. Conforme delineado por Wasserman e Faust (1994), expressa o número de laços adjacentes que um conselheiro possui com outros conselheiros da mesma rede de forma a permitir a avaliação dos efeitos dos laços diretos constituídos. Essa medida foi expressa de forma percentual e representa a razão entre o número de laços entre conselhos de administração, menos 1 (um), ponderado pelo total de laços possíveis na rede (MENDES-DA-SILVA et al, 2008).

A variável de centralidade da intermediação (Betweeness) capta os laços indiretos (não adjacentes) formados pelas interações entre dois conselheiros (WASSERMAN; FAUST 1994). A centralidade de intermediação é uma medida baseada no número de caminhos mínimos entre vértices que passam por um determinado ponto da rede. Portanto, quanto mais central o vertíce, mais caminhos passarão por ele (GABARDO, 2015). Essa medida foi obtida de forma percentual a partir da razão entre o número de caminhos mínimos entre os vértices $i$ e $j$ que passam pelo vertíce $v$, e o número de todos os caminhos mínimos entre $i$ e $j$.

A variável de qualidade dos laços (Holes) identifica a capacidade dos conselhos de administração em receber informações, recursos e conhecimento por meio de seus contatos. Conforme Burt (2004) dada duas redes $X$ e $Y$ com o mesmo número de conexões, mas onde as conexões da rede $Y$ são dentro do grupo e as conexões da rede $X$ são com nós fora do grupo imediato, a rede $X$ receberá maior fluxo de informação inédita em razão da existência de Structural Holes. Portanto, quanto menor o número de laços redundantes, maior o número de lacunas estruturais e, por conseguinte, os laços formados nessas lacunas têm menor redundância de informação. Para o cálculo da métrica de qualidade dos laços, considera-se que cada conselheiro apresenta $n$ número de contatos. Os laços redundantes podem ser mensurados pela razão entre a presença de laços duplicados $(2 l)$ referentes ao mesmo contato e o número total de contatos $(n)$. Para calcular o número de laços não redundantes é preciso subtrair do número total de conexões, os laços reduntantes. Por fim,

os laços não redundantes entre conselhos de administração são divididos pelo número de laços da rede, ponderado, assim, a métrica em percentuais. (MENDES-DA-SILVA et al, 2008).

Foram incluídas no modelo, ainda, as variáveis de controle (i) Rentabilidade do ativo (ROA), obtida pela razão entre o lucro líquido e o ativo total da empresa; e (ii) Alavancagem Contábil (ALAV), obtida pela razão entre as obrigações de curto e longo prazo e o ativo total da empresa. As empresas mais rentáveis tendem a sinalizar tal condição como forma de reduzir o risco de seleção adversa (CAMPBELL; SHRIVES; BOHMBACK-SAAGER, 2001; YAMAMOTO; SALOTTI, 2006), pois buscam mostrar aos investidores atuais e potenciais que 
possuem vantagens competitivas. Em relação à alavancagem contábil, Depoers (2000) comenta que as empresas com maior proporção da dívida na estrutura de capital tendem a divulgar mais informações na tentativa de demonstrar que estão efetivamente utilizando os recursos da melhor forma.

A amostra é aleatória e compreende 70 empresas não financeiras que mantiveram o registro ativo na BM\&FBovespa para todo o período de 2010 a 2015, obtendo-se um painel balanceado com 420 observações. O número de empresas foi calculado admitindo-se estimar a verdadeira percentagem populacional com $5 \%$ de significância e margem de erro de $10 \%$. A população considerada foi de 297 empresas com registro ativo, já excluídas as empresas de negócios financeiros, como seguradoras e bancos, sendo esta uma alternativa para não prejudicar a compreensão de algumas variáveis utilizadas no estudo.

A coleta das informações para compor a variável IDV foi realizada de forma manual com base no alinhamento do escopo do item da métrica ao conteúdo das sentenças em seu contexto (CONSONI; COLAUTO, 2016). Este procedimento foi iniciado no Relatório da Administração e finalizado com as informações disponibilizadas em alguns campos do Formulário de Referência, sempre respeitando essa ordem. Cada uma das medidas de rede e, consequentemente, caracterização do board interlocks, foi calculada para cada empresa-ano com o uso do software Ucinet ${ }^{\circledR} 6.0$ para Windows.

Para a obtenção das métricas do board interlocks foram coletadas anualmente as informações referentes aos membros do conselho de administração e diretores de cada empresa da amostra. Essas informações foram obtidas a partir dos Formulários de Referência publicados no site da Comissão de Valores Mobiliários (CVM). Após a coleta, foram identificadas manualmente as conexões entre as empresas, a partir, do software Microsoft Excel $^{\mathbb{R}}$. Na sequência, para cada ano, foi elaborada uma matriz evidenciando as ligações existentes entre as empresas, essas ligações foram exportadas para o software Ucinet ${ }^{\circledR} 6.0$ para Windows e obtidas métricas de análise de redes sociais. As demais variáveis do modelo (Equação 1) foram obtidas na Plataforma Bloomberg ${ }^{\circledR}$.

\section{Análise e Discussão}

As conexões estabelecidas entre conselhos de administração de empresas da amostra são apresentadas na Figura 1. A configuração da rede é alterada ao longo do período sem, contudo, afetar substancialmente a posição central que algumas empresas ocupavam na rede.

Figura 1: Formação do board interlocks no período de 2010 a 2015
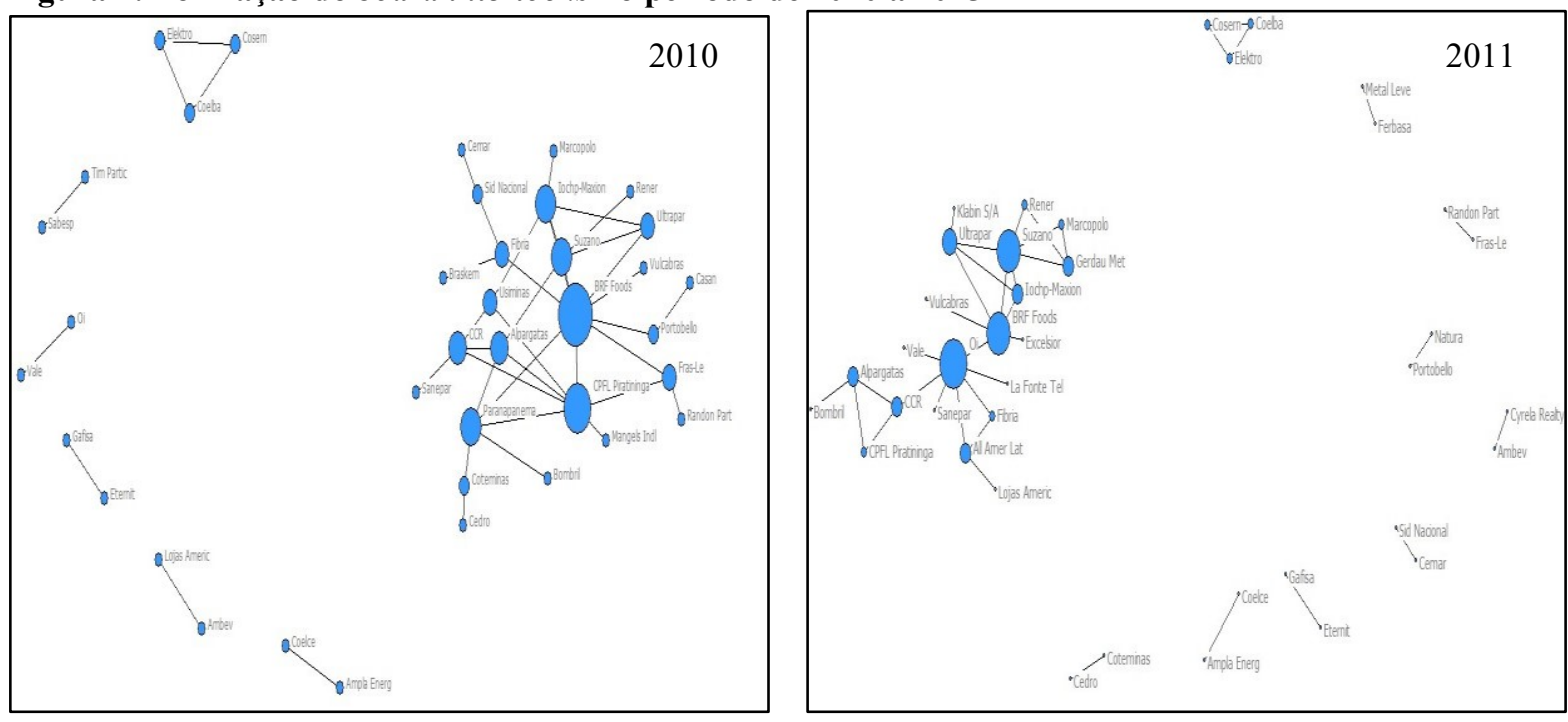

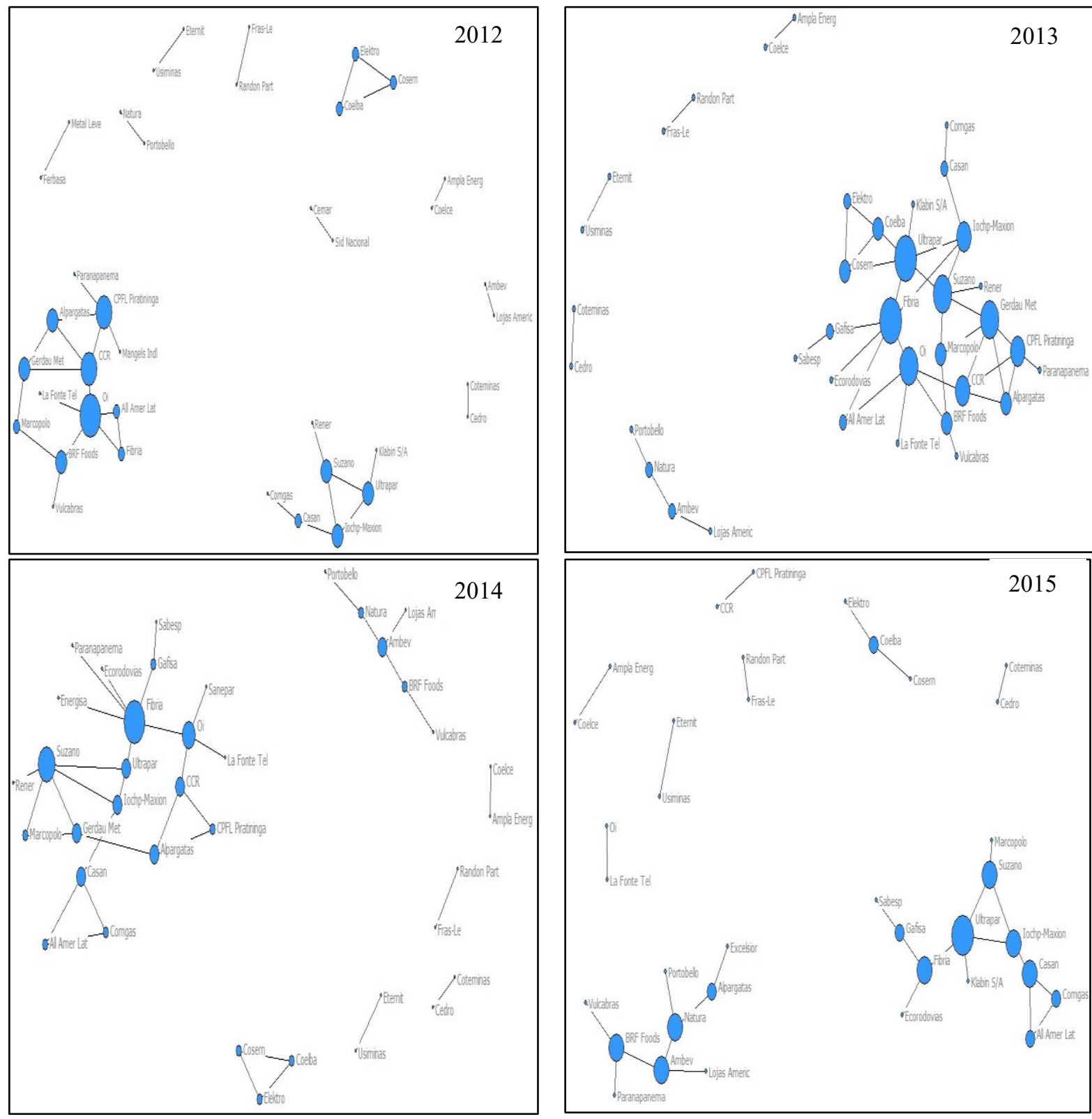

Fonte: Elaborado pelos autores

Em 2010 nota-se que as companhias com mais conexões diretas são a Ampla Energia e a Coelce com onze membros do conselho em comum. Na sequência Coelba, Consern e CPFL Piratininga (10 membros), BRF Foods (9 membros), CCR (8 membros), Alpargatas e Paranapanema (6 membros) e Oichp-Maxion e Suzano (5 membros). É interessante observar que embora a Ampla Energia compartilhe onze membros do conselho, esses membros estão ligados a Coelce, companhia do mesmo grupo econômico. É diferente, por exemplo, da BRF Foods na qual as nove ligações estão estabelecidas em conselhos de diferentes empresas.

Outro aspecto observado refere-se às conexões estáveis e temporárias. A maioria das que ocupam posições centrais na rede mantém, em geral, esse posto com pequenas alterações no número de laços. A empresa OI SA apresentou entre os anos de 2011 e 2014 muitas ligações; em 2011 foram 10 conexões com os demais conselhos da amostra, porém desfeitas nos anos subsequentes, resistindo apenas uma ligação.

$\mathrm{Na}$ Tabela 1 apresenta-se a estatística descritiva relacionada ao comportamento das variáveis ao longo do período. Verifica-se que as variáveis depentendes não apresentam indícios de multicolineariedade (FIV $<5)$. A variável IDV compreende valores entre 0 e 1 e, 
em média, a divulgação das empresas ficou abaixo de $50 \%$ dos itens da métrica, sendo a variabilidade desse índice de 37\% em relação à média, o que demostra certa heterogeneidade entre empresas. Muitas empresas não provêm informações para a maioria dos itens da métrica, direcionando para conteúdos específicos, especialmente, aqueles sobre desempenho econômico-financeiro, semelhante à descrição feita por Consoni e Colauto (2016). As variáveis de controle mostram que as empresas da amostra apresentaram, em média, rentabilidade (ROA) de $5,14 \%$ sobre ativo total e que possuem estrutura de capital composta predominantemente por capital de terceiros (ALAV), o qual, em média, representa 58\% das origens de recursos.

Tabela 1 Análise descritiva das variáveis

\begin{tabular}{l|c|c|c|c}
\hline Variáveis & Média & Desvio Padrão & CV & FIV \\
\hline IDV & 0,46 & 0,17 & 0,37 & - \\
Degree & 0,23 & 0,38 & 1,65 & 1,15 \\
Betweeness & 0,01 & 0,05 & 5,00 & 1,11 \\
Holes & 0,36 & 0,48 & 1,33 & 1,04 \\
ROA & 5,14 & 10,90 & 2,12 & 1,07 \\
ALAV & 0,58 & 0,33 & 0,57 & 1,04 \\
\hline
\end{tabular}

Nota: IDV = índice de divulgação voluntária; Degree = centralidade de grau; Betweeness $=$ centralidade de intermediação; Holes = ligações não redundantes; ROA = razão entre o lucro líquido e o ativo total; ALAV = razão entre as obrigações de curto e longo prazo e o ativo total; CV é o coeficiente de variação; FIV é o fator de inflação da variância.

Fonte: Elaborado pelos autores.

As variáveis de centralidade demonstram que as empresas da amostra apresentam, em média, um baixo nível de ligações diretas e indiretas e, portanto, sinaliza que há empresas com pouca ou nenhuma conexão. Por outro lado, as redes formadas foram ricas em conexões não redundantes. As medidas relacionadas à configuração das redes apresentaram alta variabilidade.

Tabela 2 Matriz de correlação das variáveis

\begin{tabular}{l|c|c|c|c|c|c}
\hline & IDV & Degree & Betweeness & Holes & ROA & ALAV \\
\hline IDV & 1 & & & & & \\
\hline Degree & $0,185^{* * *}$ & 1 & & & & \\
\hline Betweeness & $0,091^{*}$ & $0,280^{* * *}$ & 1 & & & \\
\hline Holes & $0,318^{* * *}$ & $0,575^{* * *}$ & $0,582^{* * *}$ & 1 & & \\
\hline ROA & $0,255^{* * *}$ & $0,166^{* * *}$ & $-0,007$ & 0,062 & 1 & \\
\hline ALAV & 0,064 & 0,033 & 0,005 & 0,040 & $-0,161^{* * *}$ & 1 \\
\hline
\end{tabular}

Nota: IDV = índice de divulgação voluntária da empresa $i$ no ano $t$; Degree = centralidade de grau da empresa $i$ no ano $t$; Betweeness = centralidade de intermediação da empresa $i$ no ano $t$; Holes $=$ ligações não redundantes da empresa $i$ no ano $t$; ROA $=$ razão entre o lucro líquido e o ativo total da empresa $i$ no ano $t$; ALAV $=$ razão entre as obrigações de curto e longo prazo e o ativo total da empresa $i$ no ano $t$. Razão $t$ entre colchetes. *, **, ***: significativo a 10,5 e $1 \%$.

Fonte: elaborado pelos autores.

A correlação entre as variáveis (Tabela 2) indica uma relação positiva e significativa entre IDV, conexões formadas e a rentabilidade das companhias. Esses resultados sugerem que, a priori, há indicios que a divulgação voluntária pode ser fixada pelas empresas a partir das ligações diretas, bem como, pela qualidade dos laços. Ademais, que os maiores índices de divulgação estão presentes entre empresas de maior rentabilidade, o que talvez justifique a presença de conteúdo predominantemente sobre análise econômico-financeira quando avaliados os relatórios de administração e formulário de referência na amostra. Ou seja, as empresas buscam comunicar resultados positivos com ênfase na distinção de sua performance.

A relação entre board interlocks e divulgação voluntária foi determinada pelo modelo de regressão de dados em painel, conforme enunciado pelas hipóteses $\mathrm{H}_{1}, \mathrm{H}_{2} \mathrm{e} \mathrm{H}_{3}$, da seção 2 deste artigo. Os resultados estão apresentados na Tabela 3. 
Tabela 3 Modelo de dados em painel para a variável dependente IDV

\begin{tabular}{lccc}
\hline Variáveis & $\begin{array}{c}\text { Modelo 1 } \\
\text { Pooled }\end{array}$ & $\begin{array}{c}\text { Modelo 2 } \\
\text { Efeitos Fixos }\end{array}$ & $\begin{array}{c}\text { Modelo 3 } \\
\text { Efeitos Aleatórios }\end{array}$ \\
\hline Constante & $0,3751^{* * *}$ & $0,3752^{* * *}$ & $0,3749^{* * *}$ \\
{$\left[\begin{array}{l}{[19,00]} \\
\text { Degree }\end{array}\right.$} & $0,0402^{* * *}$ & {$[20,64]$} & $0,0379^{*}$ \\
\hline Betweeness & {$[2,595]$} & 0,0127 & {$[1,740]$} \\
\hline Holes & $0,2873^{* * *}$ & {$[0,661]$} & $0,2888^{*}$ \\
\hline ROA & {$[2,722]$} & $0,3202^{* * *}$ & {$[1,944]$} \\
\hline ALAV & $0,8007^{* * *}$ & {$[3,076]$} & $0,0796^{* * *}$ \\
\hline R-quadrado & {$[5,558]$} & $0,0706^{* * *}$ & {$[4,934]$} \\
F de Fisher & $0,0036^{* * *}$ & {$[4,835]$} & $0,0035^{* * *}$ \\
Akaike (CIA) & {$[3,725]$} & $0,0030^{* * *}$ & $0,04699^{* *}$ \\
Schwarz (CIS) & $0,0445^{*}$ & {$[3,014]$} & {$[1,979]$} \\
Observações & {$[1,868]$} & $0,0663 * *$ & - \\
\hline Testes de diagnóticos de painel & 0,1398 & {$[2,505]$} & - \\
\hline Teste F & $14,62^{* * *}$ & $2,33^{* * *}$ & $-362,44$ \\
p-valor & $-362,48$ & $-327,04$ & $-338,19$ \\
\hline Teste Breusch-Began & $-338,24$ & $-23,98$ & 450 \\
p-valor & 450 & 450 & \\
\hline Teste Hausmann & 1,3823 & & \\
p-valor & 0,0331 & & \\
\hline Nota: IDV & 2,6024 & & \\
\hline
\end{tabular}

Nota: IDV = índice de divulgação voluntária da empresa $i$ no ano $t$; Degree = centralidade de grau da empresa $i$ no ano $t$; Betweeness $=$ centralidade de intermediação da empresa $i$ no ano $t$; Holes $=$ ligações não redundantes da empresa $i$ no ano $t$; ROA = razão entre o lucro líquido e o ativo total da empresa $i$ no ano $t$; ALAV $=$ razão entre as obrigações de curto e longo prazo e o ativo total da empresa $i$ no ano $t$. Razão $t$ entre colchetes. *, **, ***: significativo a 10,5 e $1 \%$.

Fonte: elaborado pelos autores.

Para cada uma das hipóteses $\left(\mathrm{H}_{1}, \mathrm{H}_{2}\right.$ e $\left.\mathrm{H}_{3}\right)$ foram considerados diferentes elementos constitutivos de centralidade das redes sociais (degree, betweeness e structural holes, respectivamente). Presume-se que tais elementos constitutivos estejam relacionados positivamente com o índice de divulgação volutnária das empresas.

Os testes de diagnósticos de painel, parte inferior da Tabela 3, indicam que, ao nível de significância de 1\%, o modelo Pooled (Modelo1) é adequado à análise. Esse modelo indica que no período de análise a variação do índice de divulgação voluntária ocorre predominantemente entre empresas, mantendo-se mais ou menos constante para a empresa ao longo do tempo. Em outras palavras, pode-se entender que cada empresa determinou o conjunto ideal de informação a ser divulgada voluntariamente e não se distanciou consideravelmente desse limite.

A partir da interpretação dos resultados do modelo Pooled infere-se que a presença de ligações diretas (degree), ligações indiretas (betweeness) e ligações não redundantes (Holes) estão fortemente relacionadas com maiores índices de divulgação voluntária de informações econômicas e financeiras, permitindo não rejeitar as três hipóteses formuladas. Resultados semelhantes são apresentados por Braam e Borghans (2014) e Chan et al. (2017), os quais sugerem que o board interlocks é um importante canal de comunicação de diversas práticas corporativas e, nesse sentido, tende a influenciar positivamente a prática da divulgação voluntária. Ainda, a relação entre o board interlocks e a divulgação voluntária apresenta-se consistente para empresas com maior rentabilidade e endividamento, uma vez que, a literatura entende que tais condições incentivariam as empresas a praticar a divulgação voluntária. 
Em conjunto, isto mostra que a estrutura das redes formadas pelas empresas da amostra favorece o compartilhamento de práticas de divulgação voluntária, não só por pertencerem ao mesmo grupo econômico, mas, principalmente por apresentarem um nível de relacionamento de "qualidade", medida pela centralidade de informação, ou seja, não redundância de ligações na formação das conexões. Tais características, como estabelece a Teoria da Dependência de Recursos, facilitariam o gerenciamento e o controle do fluxo de recursos, como por exemplo, ao reduzir incertezas sobre consequências econômicas da divulgação voluntária.

Nesse sentido, a maior incidência de divulgação voluntária nos relatórios anuais em empresas interligadas pelo Conselho de Administração pode ser resultado da troca de experiências entre os profissionais do conselho, ou seja, este efeito seria uma resposta das empresas às expectativas e pressões externas. Supõe-se que o compartilhamento de experiências aumentaria a capacidade do Conselho de Administração de compreender as consequências econômicas da divulgação e de suas implicações na avaliação da empresa. Este fenômeno é relativamente importante em mercados em desenvolvimento como o brasileiro, uma vez que os incentivos para a divulgação voluntária, como expõem Ball, Robin, e Wu (2003), Liang, Lin, e Chin (2012) e Leuz e Verrecchia (2000), são relativamente fracos devido à baixa demanda por alta qualidade e transparência nos relatórios publicados.

\section{Conclusões}

A separação entre propriedade e controle levou ao estabelecimento de mecanismos de governança corporativa que objetivam responder adequadamente às expectativas dos provedores de capital e de outras potenciais fontes de pressão. Embora as empresas possam recorrer a vários mecanismos internos de governança corporativa, a literatura admite ser o conselho de administração um dos mais influentes para atingir um alto nível de transparência. Para Cheng e Courtenay (2006), o conselho de administração assegura a confiabilidade das informações, pois exige receber relatórios periódicos dos gestores como forma de acompanhar as operações da empresa. Assim, o conselho de administração fornece um "pacote de monitoramento interno", que reduz a possibilidade de os gestores se envolverem em comportamentos de autoserviço, ao mesmo tempo, forçando-os a divulgar informação adicional.

Nos últimos anos, a Teoria da Dependência de Recursos tem destacado que as conexões entre os profissionais do Conselho de Administração seriam um meio de as empresas reduzirem incertezas do ambiente, pois o board interlocks facilitaria o gerenciamento e o controle do fluxo dos mais variados recursos. A informação é entendida, assim, como um recurso de consequências econômicas incertas, sendo o trade-off entre o custo e o benefício de se divulgar volutnariamente determinada informação fixado com mais segurança por meio do compartilhamento de experiências entre profisisonais dos conselhos. A lógica é que as empresas estão mais inclinadas a adotarem determinadas práticas organizacionais quando compartilham conselheiros de empresas onde tal prática já é recorrente.

Nesse contexto, investigou-se a influência da formação do board interlocks na prática de divulgação voluntária nos relatórios anuais de empresas não financeiras listadas na BM\&FBovespa, atualmente, B3 Brasil, Bolsa, Balcão, abrangendo o período de 2010 a 2015 . A estrutura teórica sugere que em economias de mercado, caracterizadas pela incerteza e competição por fundos, as empresas interligadas por meio dos profissionais do conselho de administração são mais propensas a aumentar a divulgação voluntária nos relatórios anuais. $\mathrm{O}$ aumento ocorre porque a experiência obtida em outros conselhos seria compartilhada de modo a facilitar a compreensão das expectativas e pressões externas e, consequentemente, da forma como as escolhas estratégicas relacionadas à divulgação de informações afetariam a avaliação da empresa. 
De acordo com as expectativas teóricas, os resultados não permitiram rejeitar as três hipóteses estabecidas neste estudo, mostrando que a presença de ligações diretas (degree), ligações indiretas (betweeness) e ligações não redundantes (structural holes) estão significativamente relacionadas com maiores índices de divulgação voluntária de informações econômicas e financeiras nos relatórios anuais das empresas da amostra. Desta forma, enfatizase a influência das relações intraorganizacionais e interpessoais, além das características corporativas na explicação das práticas de divulgação.

Destaca-se que os resultados podem estar condicionados à amostra, o que limita a generalização dos mesmos, e à ideia de que as decisões de divulgação possam ser afetadas de maneira uniforme apenas pelo board interlocks. Aliás, Braam e Borghans (2014), chamam a atenção de que não só o board interlocks pode exercer influência nas estratégias informacionais, uma vez que coalizões dominantes dentro da empresa poderiam, até mesmo, ser mais influentes para controlar essas estratégias. Todavia, a formação do board interlocks apresenta-se neste estudo como um fator de disseminação das melhores práticas de divulgação voluntária. O índice de divulgação voluntária global é maior em empresas com presença de estrutura de redes entre conselheiros na qual a empresa está inserida expondo um fator, de uma série de fatores potenciais que induzem as práticas de divulgação voluntária no Brasil. Em outras palavras, o comportamento da divulgação voluntária nesse ambiente institucional não é determinado somente pelas características da empresa e dos setores de atuação dentre outras, mas também pela influência de estruturas sociais.

\section{Referências}

ADAMS, Renée B.; HERMALIN, Benjamin E; WEISBACH, Michael S. The Role of Boards of Directors in Corporate Governance: A Conceptual Framework and Survey. Journal of Economic Literature, v. 48, n. 1, p. 58-107, 2010. doi: 10.1257/jel.48.1.58

AMBROZINI, Luciana Siqueira. Pressões Internas e Externas na Utilização de Padrões de Divulgação de Informações Socioambientais Amplamente Aceitos: Uma Análise sobre Estrutura Corporativa, Ambiente Institucional e Perspectiva da Legitimação. Revista Contemporânea de Contabilidade, v. 14, n. 31, p. 03-25, 2017. doi.org/10.5007/2175$8069.2017 \mathrm{v} 14 \mathrm{n} 31 \mathrm{p} 3$

ANDERSON, Ronald C.; MANSI, Sattar A.; REEB, David M. Board Characteristics, Accounting Report Integrity, and the Cost of Debt. Journal of Accounting and Economics, v. 37, n. 3, p. 315-342, 2004. doi.org/10.1016/j.jacceco.2004.01.004

AU, Kevin; PENG, Mike W.; WANG, Denis. Interlocking Directorates, Firm Strategies, and Performance in Hong Kong: Towards a Research Agenda. Asia Pacific Journal of Management, v.17, p. 29-47, 2000. doi.org/10.1023/A:1015432819596

BALL, Ray; ROBIN, Ashok; WU, Joanna Shuang. Incentives versus Standards: Properties of Accounting Income in Four East Asian Countries. Journal of Accounting and Economics, v. 36, n. 1-2, 235-270, 2003. doi.org/10.1016/j.jacceco.2003.10.003

BECKMAN, Christine M.; HAUNSCHILD, Pamela R.; PHILIPS, Damon J. Friends or Strangers? Firm Specific Uncertainty, Market Uncertainty and Network Partner Selection. Organization Science, v. 15, n. 3, p. 259-275, 2004. doi.org/10.1287/ orsc. 1040.0065

BERTOMEU, Jeremy; BEYER, Anne; DYE, Ronald A. Capital Structure, Cost of Capital, and Voluntary Disclosures. The Accounting Review, v. 86, n. 3, p. 857-886, 2011. doi.org/10.2308/accr.00000037 
BEYER, Anne; COHEN, Daniel A; LYS, Thomas Z.; WALTHER, Beverly R. The Financial Reporting Environment: Review of the Recent Literature. Journal of Accounting and Economics, v. 50, n. 2-3, p. 296-343, 2010. doi.org/10.1016/j.jacceco.2010.10.003

BORGATTI, Stephen; CROSS, Rob. A Relational View of Information Seeking and Learning in Social Networks. Management Science, v. 49, n. 4, p. 432-445, 2003. doi.org/10.1287/mnsc.49.4.432.14428

BRAAM, Geert; BORGHANS, Lex. Board and Auditor Interlocks and Voluntary Disclosure in Annual Reports. Journal of Financial Reporting and Accounting, v. 12, n. 2, p. 135160, 2014. doi.org/10.1108/JFRA-11-2012-0054

BRANDÃO, Isac de Freitas; ASSUNÇÃO, Renata Rouquayrol; PONTE, Vera Maria R.; REBOUÇAS, Sílvia Maria Dias Pedro. Fatores Determinantes do Guidance das Companhias Listadas na BM\&FBovespa. Revista Contemporânea de Contabilidade, v. 10, n. 21, p. 87114, 2013. doi.org/10.5007/2175-8069.2013v10n21p87

BURT, Ronald S. Cooptive Corporate Actor Networks: A Reconsideration of Interlocking Directorates Involving American Manufacturing. Administrative Science Quarterly, v. 25, n.4, p. 557-581, 1980. doi:10.2307/2392281

BURT, Ronald S. Structural Holes and Good Ideas. American Journal of Sociology, v. 110, n. 2, p. 349-399, 2004. doi: 10.1086/421787

CAI, Ye; DHALIWAL, Dan S.; KUI, Yongtae; PAN, Carrie. Boards Interlocks and the Diffusion Disclosure Policy. Review of Accounting Studies, v. 19, n. 3, p. 1186-1119, 2014. doi.org/10.1007/s11142-014-9280-0

CAMPBELL, David; SHRIVES, Philip; BOHMBACH-SAAGAR, Heike. Voluntary Disclosure of Mission Statements in Corporate Annual Reports: Signaling What and Do Whom? Business and Society Review, v. 106, n. 1, p. 65-87, 2001. doi.org/10.1111/00453609.00102

CARPENTER, Mason A.; WESTPHAL, James D. The Strategic Context of External Network Ties: Examining the Impact of Director Appointments on Board Involvement in Strategic Decision Making. Academy of Management Journal, v. 44, n. 4, p. 639-660, 2001. doi.org/10.5465/3069408

CHAN, Ann Ling-Ching; LEE, Edward; PETAIBANLUE, Jirada; TAN, Ning. Do Board Interlocks Motivate Voluntary Disclosure? Evidence from Taiwan. Review of Quantitative Finance Accounting, v. 48, n. 2, p. 441-466, 2017. doi.org/10.1007/s11156-016-0557-1

CHENG, Eugene C. M.; COURTENAY, Stephen M. Board composition, regulatory regime and voluntary disclosure. The International Journal of Accounting, v. 41, p. 262-289, 2006. doi.org/10.1016/j.intacc.2006.07.001

COLEMAN, James S. Social Capital in the Creation of Human Capital. The American Journal of Sociology, v. 94, p. S95-S120, 1988. doi.org/10.1086/228943

CONNELLY, Brian L.; SLYKE, Erik J. Van. The Power and Peril of Board Interlocks. Organizational Performance, v. 55, p. 403-408, 2012. doi.org/10.1016/j.bushor.2012.03.006

CONSONI, Silvia; COLAUTO, Romualdo Douglas. Voluntary Disclosure in the Context of Convergence with International Accounting Standards in Brazil. Review of Business Management, v. 18, n. 62, p. 658-677, 2016. doi.org/10.7819/rbgn.v18i62.2242 
CORE, John E. A Review of the Empirical Disclosure Literature: Discussion. Journal of Accounting and Economics, v. 31, n. 1-3, p. 441-456, 2001. doi.org/10.1016/S01654101(01)00036-2

CVM - COMISSÃO DE VALORES MOBILIÁRIOS. Recomendações da CVM sobre governança corporativa. p. 1-13. 2002. Disponível em:

<http://www.cvm.gov.br/export/sites/cvm/decisoes/anexos/0001/3935.pdf>. Acesso em: 01 mar. 2019.

DALZIEL, Thomas; GENTRY, Richard J.; BOWERMAN, Michael. An Integrated AgencyResource Dependence View of the Influence of Directors' Human and Relational Capital on Firms' R\&D Spending. Journal of Management Studies, v. 48, n. 6, p. 1217-1242, 2011. doi.org/10.1111/j.1467-6486.2010.01003.x

DEPOERS, Florence. A Cost-Benefit Study of Voluntary Disclosure: Some Empirical Evidence form French Listed Companies. European Accounting Review, v. 9, n. 2, p. 245263, 2000. doi.org/10.1080/09638180050129891

DYE, Ronald A. An Evaluation of "Essays on Disclosure" and the Disclosure Literature in Accounting. Journal of Accounting \& Economics, v. 32, p. 181-135, 2001. doi.org/10.1016/S0165-4101(01)00024-6

DYE, Ronald A. Proprietary and Nonproprietary Disclosures. Journal of Business, v. 59, n. 2; p. 331-366, 1986. doi.org/10.1086/296331

FICH, Eliezer M.; SHIVDASANI, Anil. Are Busy Boards Effective Monitors? The Journal of Finance, v. 61, n. 2, p. 689-724, 2006. doi.org/10.1111/j.1540-6261.2006.00852.x

FICH, Eliezer M.; WHITE, Lawrence J. Why do CEOs reciprocally sit on each other's boards? Journal of Corporate Finance, v. 11, n. 1-2, 175-195, 2005.

doi.org/10.1016/j.jcorpfin.2003.06.002

FRACASSI, Cesare; TATE, Geoffrey. External networking and internal firm governance.

The Journal of Finance, v. 67, n.1, p.153-194. 2012. doi.org/10.1111/j.15406261.2011.01706.x

GABARDO, Ademir C. Análise de redes sociais: uma visão computacional. Novatec: São Paulo, 2015.

GARCÍA-MECA, Emma; SÁNCHEZ-BALLESTA, Juan P. The Association of Board Independence and Ownership Concentration with Voluntary Disclosure: A Meta-Analysis. European Accounting Review, v. 19, n. 3, p. 603-627, 2010. doi.org/10.1080/09638180.2010.496979

GIBBINS, Michael; RICHARDSON, Alan; WATERHOUSE, John. The Management of Corporate Financial Disclosure: Opportunism, Ritualism, Policies, and Process. Journal of Accounting Research, v. 28, n. 1, p. 121-143, 1990. doi: 10.2307/2491219

GOYAL, Sanjeev; VEGA-REDONDO, Fernando. Structural Holes in Social Networks. Journal of Economic Theory, v.137, p. 460 - 492, 2004. doi.org/10.1016/j.jet.2007.01.006

GRANOVETTER, Mark S. The Strength of Weak Ties. American Journal of Sociology, v. 78, N. 6, p. 1360-1380, 1973. doi.org/10.1086/225469

HAUNSCHILD, Pamela R. Interorganizational Imitation: The Impact of Interlocks on Corporate Acquisition Activity. Administrative Science Quarterly, v. 38, n. 4, p. 564-592, 1993. doi.org/10.2307/2393337 
HAUNSCHILD, Pamela R.; BECKMAN, Christine M. When Do Interlocks Matter? Alternate Sources of Information and Interlock Influence. Administrative Science Quarterly, v. 43, n. 4, p. $815-844$, 1998. doi: 10.2307/2393617

HAYNES, Katalin Takacs; HILLMAN, Amy. The Effect of Board Capital and CEO Power on Strategic Change. Strategic Management Journal, v. 31, p. 1145-1163, 2010. doi.org/10.1002/smj.859

HEALY, Paul M.; PALEPU, Krishna G. Information Asymmetry, Corporate Disclosure and Capital Markets: A Review of Empirical Disclosure Literature. Journal of Accounting and Economics, v.31, n. 1-3, p. 405-440, 2001. doi.org/10.1016/S0165-4101(01)00018-0

HILLMAN, Amy J.; CANNELLA, Albert A.; PAETZOLD, Ramona L. The Resource Dependence Role of Corporate Directors: Strategic Adaptation of Board Composition in Response to Environmental Change. Journal of Management Studies, v. 37, n. 2, p. 235 256, 2000. doi.org/10.1111/1467-6486.00179

HILLMAN, Amy J.; DALZIEL, Thomas. Boards of Directors and Firm Performance: Integrating Agency and Resource Dependence Perspectives. Academy of Management Review, v. 28, n. 3, p. 383-396, 2003. doi.org/10.2307/30040728

HILLMAN, Amy J.; WITHERS, Michael C.; COLLINS, Brian J. Resource Dependence Theory: A Review. Journal of Management, v. 35, n.6, p. 1404-1427, 2009. doi.org/10.1177/0149206309343469

HUNG, Humphry. A Typology of the Theories of the Roles of Governing Boards. Corporate Governance: An International Review, v. 6, n. 2, p. 101-111, 1998. doi.org/10.1111/14678683.00089

IBGC - INSTITUTO BRASILEIRO DE GOVERNANÇA CORPORATIVA. Code of Best Practices of Corporate Governance. $5^{\text {a }}$ Edição. 2015. Disponível em: $<$ https://conhecimento.ibgc.org.br/Paginas/Publicacao.aspx?PubId=23610 $>$. Acesso em: 01 mar. 2019.

KLAPPER, Leora F.; LOVE, Inessa. Corporate Governance, Investor Protection and Performance in Emerging Markets. Journal of Corporate Finance, v. 10, p. 703-728, 2004. doi.org/10.1016/S0929-1199(03)00046-4

LA PORTA, Rafael; LOPEZ-DE-SILANES, Florencio; SHLEIFER, Andrei; VISHNY, Robert W. Law and Finance. Journal of Political Economy, v. 106, n. 6, p. 1113-1155, 1998. doi: $10.1086 / 250042$

LAZZARINI, Sergio G. Mudar Tudo para não Mudar Nada: Análise da Dinâmica de Redes de Proprietários no Brasil como "Mundos Pequenos". RAE-eletrônica, v. 6, n. 1, p. 1-24, 2007. doi.org/10.1590/S1676-56482007000100007

LEAL, Ricardo P. C.; OLIVEIRA, Claudia L.T. de. An Evaluation of Board Practices In Brazil, Corporate Governance. The International Journal of Business in Society, v. 2, n. 3, p. 21-25, 2002. doi.org/10.1108/14720700210440053

LEUZ, Christian; NANDA, Dhananjay; WYSOCKI, Peter D. Earnings Management and Investor Protection: An International Comparison. Journal of Finance and Economics, v. 69, p. 505-527, 2003. doi.org/10.1016/S0304-405X(03)00121-1

MARTINS, Henrique Cordeiro; RODRIGUES, Suzana Braga. Atributos e Papéis dos Conselhos de Administração das Empresas Brasileiras. Revista de Administração de Empresas, v. 45, p. 23-35, 2005. doi.org/10.1590/S0034-75902005000500002 
MENDES-DA-SILVA, Wesley; ROSSONI, Luciano; MARTIN, Diógenes Leiva; MARTELANC, Roy. A Influência das Redes de Relações Corporativas no Desempenho das Empresas do Novo Mercado da Bovespa. Revista Brasileira de Finanças, v. 6, n. 3, p. 335358, 2008. Disponível em:

$<$ http://bibliotecadigital.fgv.br/ojs/index.php/rbfin/article/viewFile/1344/697>. Acesso em 01 mar. 2019.

MIZRUCHI, Mark S.; STEARNS, Linda Brewster. A longitudinal study of the formation of interlocking directorates. Administrative Science Quaterly, v. 33, n. 2, p. 194-210, 1988. doi: $10.2307 / 2393055$

MIZRUCHI, Mark. S. Análise de Redes Sociais: Avanços Recentes e Controvérsias Atuais. Rev. adm. empres., São Paulo, v. 46, n. 3, p. 72-86, Sept. 2006. Disponível em: $<$ http://www.scielo.br/scielo.php?script $=$ sci_arttext\&pid=S003475902006000300013\&lng=en\&nrm=iso>. Acesso em: 01 mar. 2019.

MIZRUCHI, Mark. S. What Do Interlocks Do? An Analysis, Critique and Assessment of Research on Interlocking Directorates. Annual Review of Sociology, v. 22, p. 271-298, 1996. doi: 10.1146/annurev.soc.22.1.271

MURCIA, Fernando Dal-Ri; SANTOS, Ariovaldo dos. Discretionary Based Disclosure: Evidence from the Brazilian Market. BAR - Brazilian Administration Review, v. 9, n. 1, p. 88-109, 2012. doi.org/10.1590/S1807-76922012000100006

ONG, Chin Huat; WAN, Tai Wai David. Board Structure, Board Process and Board Performance: A Review \& Research Agenda. Journal of Comparative International Management, v. 4, n. 1, p. 1-25, 2001. Disponível em:

$<$ https://journals.lib.unb.ca/index.php/JCIM/article/view/468/783>. Acesso em: 01 mar. 2019.

PFEFFER, Jeffrey; SALANCIK, Gerald R. The External Control of Organizations: A Resource Dependence Perspective. New York: Harper \& Row, 1978.

RIBEIRO, Flávio; COLAUTO, Romualdo Douglas. The Relationship between Board Interlocking and Income Smoothing Practices. Revista Contabilidade e Finanças, v. 27, n. 70, 55-66, 2016. doi: 10.1590/1808-057x201501320

RIBEIRO, Flávio; COLAUTO, Romualdo Douglas; CLEMENTE, Ademir. Determinants of Board Interlocking in the Brazilian Capital Market. Revista de Educação e Pesquisa em Contabilidade, v. 10, n. 4, p. 398-415, 2016. doi: 10.17524/repec.v10iX.1403

ROSSONI, Luciano; MACHADO-DA-SILVA, Clovis L. Legitimacy, Corporate Governance and Performance in BM\&FBovespa. Revista de Administração de Empresas, v. 53, n. 3, p. 272-289, 2013. doi.org/10.1590/S0034-75902013000300005

SANTOS, Rafael Liza; SILVEIRA, Alexandre di Miceli da. Board Interlocking no Brasil: A Participação de Conselheiros em Múltiplas Companhias e seu Efeito sobre o Valor da Empresa. Revista Brasileira de Finanças, v. 5, n. 2, p. 125-163, 2007.

SILVEIRA, Alexandre di Miceli da; LEAL, Ricardo Pereira Câmera; BARROS, Lucas Ayres Barreira de campos; CARVALHAL-DA-SILVA, André Luiz. Evolution and Determinants of Firms-Level Corporate Governance Quality in Brazil. Revista de Administração, v. 44, n.3, p. 173-189, 2009. Disponível em:

http://www.lti.pro.br/userfiles/downloads/Evolucao $\% 20 \mathrm{e} \% 20$ determinantes $\% 20$ qualidade $\% 2$ 0governanca\%20corporativa\%20Brasil.pdf. Acesso em: 01 mar. 2019. 
STUART, Toby E.; YIM, Soojin. Board Interlocks and the Propensity to be targeted in Private Equity Transactions. Journal of Financial Economic, v. 97, p. 174-189, 2010. doi.org/10.1016/j.jfineco.2010.03.012

SUBRAHMANYAM, A. Social Networks and Corporate Governance. European Financial Management, v. 14, n. 4, p. 633-662, 2008. doi.org/10.1111/j.1468-036X.2007.00429.x

WAGENHOFER, Alfred. Voluntary Disclosure with a Strategic Opponent. Journal of Accounting and Economics, v.12, n. 4, p. 341-363, 1990. doi.org/10.1016/01654101(90)90020-5

WASSERMAN, Stanley; FAUST, Katherine. Social Network Analysis: Methods and Applications. Cambridge: Cambridge University Press, 1994.

WESTPHAL, James D.; SEIDEL, Marc-David L.; STEWART, Katherine J. Second-Order Imitation: Uncovering Latent Effects of Board Network Ties. Administrative Science Quarterly, v. 46, n. 4, p. 717-747, 2001. doi: 10.2307/3094829

YAMAMOTO, Marina Mitiyo; SALOTTI, Bruno Meirelles. Informação Contábil: Estudos sobre a sua Divulgação no Mercado de Capitais. São Paulo: Atlas, 2006.

* Artigo apresentado no XLI Encontro da ANPAD - EnANPAD 2017 